\title{
Ventajas Competitivas de la Multinacional Agroalimentaria en Aspectos Tecnológicos
}

\author{
Oscar Alfranca \\ Ruth Rama \\ Nicholas von Tunzelmann
}

\section{Resumen}

El objetivo principal de este trabajo es analizar el funcionamiento de las carteras tecnológicas en las empresas agroalimentarias multinacionales (EMAs). Para investigar la forma en que estas empresas combinan intramuros sus diversos conocimientos técnicos, analizamos un panel de 18.611 patentes de utilidad (técnicas) y 1.359 patentes de diseño concedidas en los EEUU a 90 empresas agroalimentarias multinacionales, entre 1969 y 1994. La metodología se basa en el cálculo de correlaciones bivariadas no paramétricas entre el número de patentes de alimentos y el número de patentes concedidas a la empresa en otros 10 campos técnicos no alimentarios. Los resultados indican que la actividad patentadora en alimentos está positivamente correlacionada con la actividad patentadora en cuatro campos técnicos: Biotecnología, Química, Maquinaria y Otras patentes. Estos resultados aparecen recurrentemente en diversos grupos de empresas clasificadas por país de origen, aunque varíe la intensidad de la asociación estadística. Estas correlaciones se calculan para diversas agrupaciones muestrales y observamos que nuestros resultados son estadísticamente robustos. Finalmente, identificamos a los cinco principales innovadores en los once campos técnicos estudiados y también en innovación de diseño destinada al embalaje de los productos alimentarios.

Palabras clave: multinacionales de alimentos y bebidas; industria alimentaría; innovación; patentes.

\begin{abstract}
The main objective of this paper is to analyse patent portfolio behaviour in food and beverage multinationals (FBMs). In order to investigate the way food and beverage multinationals combine in house their various technological capabilities we analyse a panel of 18,611 utility (technical) patents and 1,359 design patents granted to such enterprises in the US during 1969-94. The methodology is based in the calculation of bivariate nonparametric correlations between the number of food patents and the number of patents granted in each of 10 non-core fields. Food patenting is positively associated with patenting in four technical fields: Biotechnology, Chemicals, Machinery and Other patents. The results are recurrent across different groups of firms classified by home-country, though the intensity of the statistical association varies. We recalculate the correlations for different sub-samples and observe that results are statistically robust. Finally, we identify the five most important innovators in the 11 technical fields and in design innovation for food packaging.
\end{abstract}

Key words: multinational food and beverage firms; food industry; innovation; patents. 


\section{INTRODUCCIÓN}

Aunque la industria agroalimentaria es, a veces, caracterizada como un sector tradicional, donde la innovación podría llegar a ser superflua (Galizzi y Venturini, 1996; Byé, 1998), ésta es actualmente un ingrediente importantísimo de la competencia entre empresas del sector (Connor, 1981). Quien hable de algunos de los aspectos más apreciados hoy día por el consumidor - calidad, seguridad, durabilidad o presentación práctica y atractiva de los alimentos - está hablando de innovación (Christensen et al., 1996).

A menudo se argumenta que, en todos los sectores industriales, una de las ventajas competitivas más relevantes de la empresa multinacional, frente a la firma nacional, es su gran capacidad de innovar (Caves, 1996). Las grandes empresas multinacionales agroalimentarias no son una excepción puesto que aglutinan más del 50\% de los inventos patentados mundialmente en alimentos y tabaco (Patel y Pavitt, 1991; Patel, 1995; Alfranca et al., 2002). Unilever, por sí sola, concentra más de la mitad de las patentes en aceites y grasas otorgadas en España, uno de los mayores productores mundiales de aceite de oliva (Rama, 1991). Puede pensarse, por lo tanto, que frente a la empresa nacional, la gran capacidad innovadora de la empresa multinacional agroalimentaria (en adelante, EMA), constituye una de las ventajas competitivas $\mathbf{O}$ (Ownership advantages) que menciona Dunning (1993) en su teoría ecléctica del negocio internacional.

En este trabajo argumentaremos que no sólo importa la cantidad de innovaciones sino también la especificidad de la cartera tecnológica de la EMA (que no debe confundirse con su cartera de productos), que le permite combinar en su propio seno diferentes tipos de conocimientos técnicos. Con pocas excepciones (von Tunzelmann, 1998), el análisis de los diversos campos técnicos en que interviene la EMA ha sido, hasta el momento, insuficientemente abordado. Sin embargo, es un aspecto de gran interés porque permite dilucidar mejor cuales son las ventajas competitivas de estas compañías. Este es un problema de gran repercusión en sistemas agroindustriales como el del Brasil, un país que es, a la vez, el mayor receptor de inversiones extranjeras agroalimentarias de América Latina, uno de los principales exportadores mundiales de alimentos y un incipiente exportador de capitales en el ámbito agroalimentario (Belik, 1994; Farina y Santos Viegas, 2004). Sus grandes empresas nacionales agroalimentarias compiten con las mayores EMAs mundiales ${ }^{(1)}$ por el enorme mercado brasileño y, potencialmente, por el de MERCOSUR. Por ello, es importante conocer en profundidad las ventajas competitivas de dichas multinacionales en el aspecto tecnológico. 
En este trabajo estudiaremos la cartera tecnológica de una muestra de 90 EMAs seleccionadas entre las 100 mayores del mundo. Utilizando una muestra de 18.611 patentes de utilidad y 1.359 patentes de diseño concedidas a dichas compañías entre 1969 y 1994, estudiaremos de qué manera dichas empresas combinan diversos tipos de innovaciones. También analizaremos, por campo técnico ${ }^{(2)}$, cuales son las más innovadoras en el sector agroalimentario multinacional.

En la sección 2 de este trabajo presentaremos los datos y metodología de la investigación. La 3 debate sus antecedentes teóricos y empíricos así como las hipótesis que pondremos a prueba. La 4 expone nuestros resultados y la 5, nuestras conclusiones.

\section{Datos y Metodología}

\section{La Muestra de Empresas}

Las 90 compañías de la muestra fueron seleccionadas a partir de AGRODATA, una base de datos sobre las 100 mayores EMAs del mundo elaborada desde los años setenta por el Institut Agronomique Méditerranéen de Montpellier (Francia) ${ }^{(3)}$. Nuestras empresas son básicamente procesadoras de alimentos pero algunas de ellas también producen productos agrícolas o se han diversificado en industrias no alimentarias. La muestra incluye 33 empresas norteamericanas, 37 europeas, 17 japonesas y 13 de otros países.

\section{La Muestra de Patentes}

Para estudiar la innovación producida por las EMAs (o sus capacidades tecnológicas) recurrimos al método del recuento de patentes. Este último tiene algunos inconvenientes bien conocidos en la bibliografía sobre el tema (Rosenberg, 1982; Archibugi y Pianta, 1992); si bien subsanables en muestras de empresas homogéneas como ésta. Sin embargo, también presenta grandes ventajas metodológicas. Las estadísticas de patentes suministran series temporales de largo plazo a escala mundial que son únicas como herramientas para el análisis de la innovación (Freeman, 1994). Por ello, son muy adecuadas para efectuar análisis de empresas procedentes de diversos países, como el que emprenderemos aquí. Por otra parte, dichas estadísticas reflejan bastante adecuadamente otros indicadores tecnológicos, como los gastos en Investigación y Desarrollo (I+D) o las actividades innovadoras de las empresas (Bound et al., 1984; Acs y Audretsch, 1989). 
En este trabajo utilizamos, como otros autores que desarrollaron comparaciones internacionales (Fagerberg, 1987; Soete, 1987), datos de patentes otorgadas a las empresas de la muestra en un solo país, los EEUU. Como demuestra Soete, las patentes concedidas en EEUU son un buen reflejo del stock mundial de tecnología.

Las 18.611 patentes de nuestra muestra, que fueron concedidas a las ya citadas 90 EMAs entre 1969 y $1994^{(4)}$, proceden del Science and Technology Policy Research (SPRU) de la Universidad de Sussex en Gran Bretaña ${ }^{(5)}$.

\section{Las Patentes no Alimentarias y la Cadena Alimentaria}

En este trabajo estudiamos los 11 campos técnicos en los cuales patentan las empresas de nuestra muestra: Agricultura, Alimentos, Biotecnología, Embalaje, Instrumentos, Maquinaria, Otros, Productos farmacéuticos y de higiene (en adelante Productos farmacéuticos), Química, Refrigeración y Tabaco ${ }^{(6)}$. En Otras se agruparon patentes referentes a textiles, papel y cartón, metales, electricidad y electrónica, vehículos, etc. Nuestras variables indican el número de patentes otorgadas a las empresas de la muestra en cada uno de los 11 campos en el período analizado. Las características de la muestra en cuanto a la distribución de las patentes según su campo técnico y el origen de las multinacionales aparecen en al Tabla 1.

Tabla 1: Características de la Muestra

Actividad Patentadora de las Principales Empresas Multinacionales Agroalimentarias, por País de Origen de la Empresa y Campo Técnico de la Patente, 1969-94 ( ${ }^{\circ}$ de patentes)

\begin{tabular}{|l|c|c|c|c|}
\hline \multicolumn{1}{|c|}{ Campo técnico } & $\begin{array}{c}\text { EMAs } \\
\text { norteamericanas }\end{array}$ & $\begin{array}{c}\text { EMAs } \\
\text { europeas }\end{array}$ & $\begin{array}{c}\text { EMAs } \\
\text { japonesas }\end{array}$ & $\begin{array}{c}\text { EMAs de } \\
\text { otros países }\end{array}$ \\
\hline Agricultura & 119 & 67 & 10 & 0 \\
\hline Biotecnología & 285 & 177 & 368 & 7 \\
\hline Química & 2269 & 1444 & 480 & 1 \\
\hline Prod farmac. & 780 & 347 & 303 & 4 \\
\hline Alimentos & 3310 & 1312 & 328 & 8 \\
\hline Instrumentos & 497 & 267 & 34 & 0 \\
\hline Maquinaria & 898 & 374 & 28 & 1 \\
\hline Otras & 2178 & 1135 & 98 & 2 \\
\hline Embalaje & 510 & 189 & 16 & 16 \\
\hline Refrigeración & 70 & 15 & 5 & 5 \\
\hline Tabaco & 638 & 35 & 1 & 0 \\
\hline TOTAL & 11554 & 5362 & 1671 & 24 \\
\hline
\end{tabular}

Fuente: elaboración propia. 
Es importante destacar que muchas de las patentes comprendidas en los campos no alimentarios citados tienen, en realidad, conexiones con la cadena alimentaria. Por ejemplo, algunos inventos consignados en Otras, como las innovaciones en electrónica, pueden ser utilizados en la planta industrial alimentaria (Christensen et al., 1996), si bien es imposible distinguirlos, con la información disponible, de otros inventos electrónicos que nada tienen que ver con ella. Del mismo modo, en Otras también se recogen inventos utilizables en las industrias de textiles o papel y cartón que pueden ser, en realidad, subproductos no alimenticios de la empresa agroalimentaria, como el almidón, los adhesivos, etc. (Gonard et al., 1991). También la empresa agroalimentaria puede producir patentes químicas vinculadas con la elaboración de ácidos orgánicos, gluconatos, sorbitol y otros productos químicos obtenidos a partir de productos agrícolas. De hecho, como señala el excelente estudio de caso de Gonard et al. (1991), las grandes empresas agroalimentarias despliegan, en este momento, un gran esfuerzo en I+D para buscar aplicaciones industriales de alto valor añadido para sus subproductos.

Las estadísticas descriptivas de las variables aparecen en la Tabla 2. El pequeño número promedio de innovaciones patentadas en ciertas especialidades sugiere que son el fruto fortuito de procesos de aprendizaje de la multinacional en ese campo, más que de un esfuerzo tecnológico sostenido con miras a la fabricación intramuros de determinados inputs o equipos. Por lo demás, un alto porcentaje de la muestra realiza actividades innovadoras en campos no alimentarios, por limitadas que éstas sean. Así, el $84 \%$ de la muestra ha obtenido al menos una patente del campo Otras y el 69\% ha conseguido alguna patente química.

Además de estas patentes de utilidad, que se refieren a aspectos técnicos de la innovación, también analizaremos, como se ha mencionado, una muestra de patentes de diseño otorgadas a las 90 empresas. Este tipo de innovación está estrechamente asociada a la innovación técnica en Alimentos (Alfranca et al., 2004) y tiene relevancia para apuntalar las marcas y el marketing de los productos.

Tabla 2: Estadísticas Descriptivas de las Variables

\begin{tabular}{|l|r|r|r|}
\hline & Media & $\begin{array}{c}\text { Desviación } \\
\text { típ. }\end{array}$ & \multicolumn{1}{c|}{ Rango } \\
\hline agricultura & 2.18 & 5.56 & 40.00 \\
biotec & 9.30 & 26.42 & 215.00 \\
química & 46.60 & 189.18 & 1427.00 \\
prod farmac & 15.93 & 73.42 & 658.00 \\
alimentos & 55.09 & 142.30 & 1117.00 \\
instrumentos & 8.87 & 27.94 & 233.00 \\
maquinaria & 14.46 & 35.11 & 194.00 \\
otras & 37.91 & 83.02 & 475.00 \\
embalaje & 7.97 & 22.84 & 145.00 \\
refrigeración & 1.00 & 3.41 & 22.00 \\
tabaco & 7.49 & 42.74 & 295.00 \\
\hline
\end{tabular}

Fuente: elaboración propia. 


\section{Antecedentes Teóricos}

En esta sección presentamos los antecedentes que nutren teóricamente nuestro trabajo de investigación, así como nuestras hipótesis.

Las grandes empresas multinacionales tienden a ser empresas multitecnológicas (Patel y Pavitt, 1997) y las EMAs no son una excepción a la regla ya que destinan una buena parte de su esfuerzo innovador a una gran variedad de campos técnicos no alimentarios, además de Alimentos y Agricultura. Un estudio de 106 grandes EMAs analizadas a lo largo de 1969-94 detecta que alrededor del 73\% de sus patentes corresponden a campos no alimentarios (von Tunzelmann, 1998). La magnitud del esfuerzo innovador de las EMAs en dichos campos se valora más claramente cuando se observa, por ejemplo, que la multinacional química dedica sólo el 29\% de sus patentes a campos técnicos ajenos a la química (Patel y Pavitt, 1997).

Ha de tenerse en cuenta que alrededor del $45 \%$ de los inventos utilizables por la industria agroalimentaria proceden de otras ciencias y técnicas, como las relativas a la química, la maquinaria, etc., originándose el restante 55\% en campos técnicos alimentarios (Rama, 1996). Sin embargo, ¿porqué las multinacionales generan ellas mismas tan alta proporción (73\%) de patentes no alimentarias?

La explicación más común es que las EMAs las destinan a sus diversos negocios, ya que muchas de estas empresas son grandes conglomerados que producen detergentes, textiles, etc., además de alimentos. Esta explicación, no obstante, es difícil de aceptar o, al menos, debe ser complementada por otras. En el grupo de las 100 mayores EMAs, las ventas de productos no alimentarios y la producción de innovaciones de ese tipo han evolucionado en forma divergente. Así, las ventas globales no alimentarias de dichas empresas han caído del 25,7\% de las ventas totales en 1981-88 a sólo 10,2\% en 1990-96 (Anastassopoulos y Rama, 2004), en gran medida como consecuencia de un cambio de estrategia planteado como vuelta al negocio de base (Ding y Caswell, 1995). Sin embargo, la proporción de las patentes no alimentarias en el total de patentes concedido a las EMAs ha crecido del 70\% del total de sus patentes en 1969-74 a casi el 77\% a mediados de los noventa (von Tunzelmann, 1998). Por otra parte, según las mismas fuentes, las empresas japonesas que son las más centradas en el agronegocio (en 199096, éste representaba el 94\% de sus ventas totales), son las que, proporcionalmente, innovan más en campos técnicos no alimentarios (79\% de las patentes de las EMAs japonesas versus 73\% en el total de EMAs).

Otra razón posible es que las EMAs produzcan ellas mismas una parte del equipo e inputs que necesitan para producir alimentos. En este orden de ideas, 
otra explicación plausible es que, aunque no produzcan dichos equipos o inputs, congruentemente con la estrategia de otras grandes empresas industriales (Patel y Pavitt, 1997), necesitan desarrollar capacidades técnicas intramuros para poder interactuar de la forma más efectiva posible con sus proveedores de tecnología. El equipamiento para la industria alimentaria, por ejemplo, necesita a menudo ser personalizado (Petroni, 2000). Otro tanto puede afirmarse respecto a muchos de los nuevos inputs utilizados por esta industria (Gonard et al., 1991). Dado que el Depto de I+D de la empresa es una herramienta no sólo para producir inventos sino también para aprender (Cohen y Levinthal, 1989), es posible que la EMA termine patentando en campos técnicos no alimentarios porque necesita desarrollar determinados conocimientos (lo que no implica, obligatoriamente, que fabrique intramuros los aparatos o inputs en cuestión). Ya desde 1979, un informe de la OCDE (1979) recalcaba el activo papel desempeñado por algunas grandes multinacionales, como Nestlé, Unilever o CPC (todas ellas en nuestra muestra), en la coordinación y la administración de la transferencia tecnológica. Para desempeñar este tipo de papel, la empresa debe desarrollar intramuros conocimientos y procesos de aprendizaje.

Como ya se señaló, otro motivo por el cual las EMAs innovan en campos no alimentarios es que producen, a partir de productos agrícolas, subproductos que venden a otros industriales (Gonard et al., 1991). Por otra parte, actualmente los procesadores de alimentos controlan cada vez más la seguridad y calidad a lo cargo de toda la cadena alimentaria, por lo cual no pocas veces pueden interesarse en inventos destinados a la producción de productos agrícolas o de inputs, particularmente cuando pueden controlar de alguna manera la producción de estos últimos a través de la integración vertical. Por ejemplo, los procesadores de patatatas pueden interesarse por aspectos biotecnológicos de la producción de estas últimas ya que sus plantas industriales están casi siempre ajustadas, al menos en los países desarrollados, a las diversas variedades de aquellas (Bijman y Enzing, 1995).

Claro está que, cualitativamente, el tipo de relación entre inventos alimentarios y no alimentarios no ha de ser el mismo en todos estos supuestos. Por definición, el conglomerado es un conjunto de diversos negocios inconexos y con escasas interrelaciones entre sus líneas productivas (Dosi et al., 1992), por lo cual es lógico argumentar que también las interrelaciones entre las capacidades técnicas de diversa índole que coexisten en su seno son escasas. Por el contrario, es mucho más lógico pensar que, en los demás ejemplos antes debatidos, ha de existir algún tipo de asociación funcional entre los patrones de innovación en alimentos y en otras especialidades técnicas.

En función del debate anterior, nos proponemos abordar las siguientes líneas de estudio: 
. La cartera tecnológica de las EMAs y, en particular, las relaciones recurrentes entre la producción de inventos alimentarios (campo técnico de Alimentos) y otro tipo de inventos no alimentarios por parte de la propia empresa.

. El grado de asociación entre diversos tipos de patentes generados por la empresa podría depender del país de origen de ésta.

. Identificamos a las EMAs más importantes según campo técnico.

\section{Resultados}

\section{Actividad Innovadora en Alimentos y en Otros Campos Técnicos}

Para cada una de las empresas de nuestra muestra, procedemos a correlacionar $^{(7)}$ su producción propia de patentes en Alimentos y en los demás campos técnicos (Tabla 3). Insistimos en que no se trata de analizar externalidades sino la producción intramuros de inventos de diferente naturaleza generados por la multinacional alimentaria. Con el propósito de identificar relaciones positivas recurrentes entre dichos tipos de inventos, subdividimos la muestra según grupos de EMAs pertenecientes a tres orígenes diferentes (Norteamérica, que incluye empresas de EEUU y Canadá; Europa y Japón).

Los resultados son los siguientes. En los cuatro casos de la Tabla 3 (es decir, el total de la muestra y los tres subtotales por país de origen), la actividad innovadora de la empresa en el campo de Alimentos está asociada con sus propias actividades innovadoras en Biotecnología, Química, Maquinaria y Otros, con una intensidad que oscila entre moderada $(\leq 0.30 \mathrm{r}>50)$ y alta $(r \geq 0.50)^{(8)}$. La asociación que se observa entre sus actividades innovadoras en Alimentos y Química y entre Alimentos y Otras es especialmente fuerte. Estos resultados no implican necesariamente una relación causal entre la actividad innovadora de la empresa en Alimentos y sus demás tipos de actividades innovadoras sino que, indican, más bien, que las compañías muy innovadoras en Alimentos también tienden a serlo en Biotecnología, Química, Maquinaria y Otras patentes. Del mismo modo, las empresas que son modestamente activas en el campo técnico de Alimentos también tienden a generar un pequeño número de inventos en los otros cuatro campos técnicos arriba citados. 


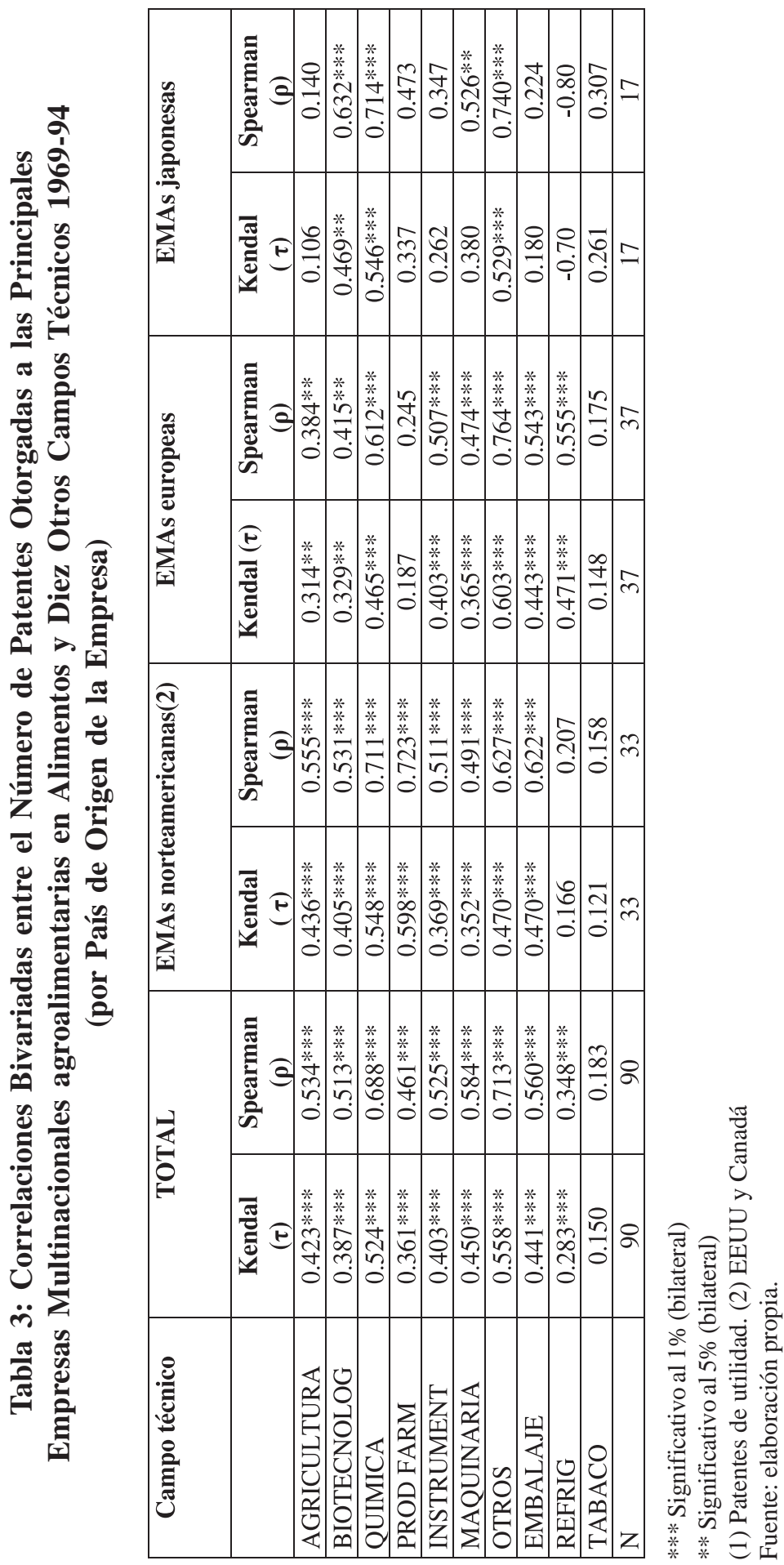


A continuación, estudiamos los gráficos de dispersión y los casos atípicos en estas asociaciones con el propósito de excluir la posibilidad de que los coeficientes de correlación obtenidos se deban a la presencia de unos pocos conglomerados (si bien, como se indica en la nota 4, se han descartado los casos más extremos porque sólo se han escogido multinacionales donde al menos el $50 \%$ de las ventas totales corresponden a agroalimentación).

\section{Análisis de Casos Atípicos}

Nos detendremos en tres campos técnicos cuya asociación con Alimentos podría levantar la sospecha de la presencia de conglomerados. Se trata de Química, Productos farmacéuticos y Otras patentes que son, además, los campos no alimentarios donde las EMAs realizan más actividades innovadoras. Estudiamos los gráficos de dispersión correspondientes (no se presentan por falta de espacio) e identificamos en cada uno de ellos a los valores atípicos en la relación del campo técnico con el de Alimentos. Luego, volvemos a calcular las correlaciones sin los casos atípicos para comprobar si se mantiene la ya observada asociación positiva en la correlación bivariada de las variables.

En el gráfico de dispersión entre Alimentos y Química, el valor atípico es Procter \& Gamble, que es efectivamente un conglomerado estadounidense. Tiene 1.427 patentes químicas y 393 de alimentos. Es un caso atípico porque la mayoría de las EMAs tiende a combinar un número mayor de patentes de Alimentos con un número reducido de patentes de Química (mientras que $P \& G$ hace lo contrario). Una vez extraído este caso, se vuelven a calcular las correlaciones bivariadas para la muestra ( $\mathrm{N}=89$ ), obteniéndose los siguientes valores: $\tau=0.514 ; \rho=0.678$, ambas estadisticamente significativas al $1 \%$.

En el gráfico de Alimentos y Otras patentes, el caso atípico es Unilever, una empresa anglo-holandesa gigantesca que posee 397 patentes de alimentos y 475 Otras patentes. También este caso es atípico porque, en general, las EMAs tienden a combinar mayores cantidades de patentes en Alimentos con una menor proporción de Otras patentes (quitando a Unilever, la media de la muestra, durante el período, es de 51 patentes de Alimentos y 33 “Otras”). Cuando se extrae el caso y se vuelven a calcular las correlaciones bivariadas para la muestra $(\mathrm{N}=$ 89), encontramos lo siguiente: $\tau=0.548 ; \rho=0.704$, ambas estadisticamente significativas al $1 \%$.

Finalmente, en el gráfico de Alimentos y Productos farmacéuticos, el caso atípico es nuevamente $\mathrm{P} \& \mathrm{G}$, con 658 patentes de productos farmacéuticos y, como se ha dicho, 393 de alimentos. Una vez más, se trata de un caso atípico porque las patentes depositadas en este campo superan a las de Alimentos. En el resto de 
las EMAs la relación se invierte pues patentan, en promedio, 51 inventos de Alimentos y sólo 9 de productos farmacéuticos. Procediendo a recalcular las correlaciones, una vez extraída P\&G, encontramos que: $\tau=0.335 ; \rho=0.441$, ambas estadisticamente significativas al $1 \%(\mathrm{~N}=89)$.

En resumen, aún extrayendo de la muestra estos casos atípicos por sus indicios de tratarse de conglomerados, comprobamos que se mantiene el signo positivo de las asociaciones y su significación estadística. Por otra parte, el análisis de la producción de Unilever, uno de los grandes innovadores en Productos farmacéuticos y Otras patentes de la muestra, es en sí mismo muy ilustrativo de las posibles sinergias tecnológicas entre la innovación en dichos campos y la cadena agroalimentaria. Así, según información de AGRODATA, la multinacional, que es líder mundial en aceites, margarinas y oleaginosos, también produce oleoquímicos, aromas, aditivos alimentarios, resinas, adhesivos, almidones y otras productos químicos o de tocador inequívocamente relacionados con la cadena agroalimentaria (Rastoin et al., 1998). En el caso de P\&G, sin embargo, la información disponible no nos ha permitido determinar si su producción no alimentaria (detergentes y otros productos) tiene vinculaciones con la cadena agroalimentaria.

Finalmente, quedaba la duda de si la positiva asociación entre las variables no se debería, en realidad, a que las modestas patentadoras de Alimentos no producen ninguna patente en los campos arriba citados. Por ello, se procedió a recalcular las correlaciones bivariadas, excluyendo los casos de empresas sin patentes de Química, Otras y Productos farmacéuticos. Una vez más volvimos a observar asociaciones positivas y estadísticamente significativas entre las variables. La nueva correlación entre Alimentos y Química tiene el siguiente resultado: $\tau=$ $0.485 ; \rho=0.653$, ambas estadisticamente significativas al $1 \%(\mathrm{~N}=84)$. La de Alimentos y Otras: $\tau=0.526 ; \rho=0.670$, ambas estadisticamente significativas al $1 \%(\mathrm{~N}=76)$. La de Alimentos y Productos farmacéuticos: $\tau=0.445 ; \rho=0.588$, ambas estadisticamente significativas al $1 \%(\mathrm{~N}=41)$. Esto confirma que nuestros resultados son robustos.

\section{Análisis por Grupos de EMAs, Según País de Origen}

Como ya se señaló, la asociación entre las citadas actividades innovadoras se confirma cuando analizamos grupos de empresas clasificadas según su origen geográfico (Tabla 3). Este es un hallazgo destacable pues prueba que nuestros resultados son estadísticamente robustos ya que las EMAs de diversos países presentan tamaños y grados de especialización en alimentos muy diferentes entre sí (Tozanli, 1998; Anastassopoulos y Rama, 2004). Por ejemplo, las EMAs estadounidenses de nuestra muestra tienen un volumen de ventas globales muy superior a las europeas y japonesas. Del mismo modo, las japonesas, como ya se 
comentó, están mucho más especializadas en la producción agroalimentaria (o menos diversificadas en otras líneas de negocio) que sus rivales occidentales.

Sin embargo, pese a la recurrencia de la asociación estadística, en algunos casos su intensidad varía según país de origen de la compañía. Por ejemplo, los coeficientes de correlación entre la actividad innovadora en Alimentos y Biotecnología son mucho más altos entre las empresas japonesas, que están posicionadas en la producción de inputs intermedios para la industria alimentaria, como productos de fermentación o aromas que se obtienen con técnicas de biotecnología (G.E.S.T., 1986). Como se verá más adelante, Ajinomoto, una multinacional de dicho origen, es la más innovadora de nuestra muestra en el campo técnico de la Biotecnología. Proporcionalmente, es muchísimo más activa en dicho campo que en el de Alimentos (mientras que las demás tienden a producir un pequeño número de patentes de Biotecnología para un número bastante superior de inventos en el campo de Alimentos). Por otra parte, la asociación entre la innovación en Alimentos y Embalaje es mucho más fuerte y estadísticamente significativa en el caso de las EMAs occidentales que en el de las japonesas, lo que podría atribuirse a que las primeras prefieren posicionarse en mercados finales de consumo (no en mercados de productos complejos intermedios, como las niponas).

Esto sugiere que los diversos grupos de compañías compiten con ventajas tecnológicas diferentes entre sí y que éstas les han permitido tomar posición en diversos mercados agroalimentarios y de ingredientes. Es posible también que cada una de las empresas despliegue capacidades técnicas específicas que las distingan de las demás en lo que se refiere a los diversos campos técnicos. Este aspecto será abordado a continuación.

\section{Principales Empresas Innovadoras}

La Tabla 4 presenta a las principales empresas innovadoras de la muestra, con indicación de su país de origen y de su participación porcentual en el total de patentes de cada campo técnico.

Algunas compañías gigantescas, como Philip Morris, Procter \& Gamble, Unilever y Nestlé, son las principales innovadoras simultáneamente en muchos de dichos campos técnicos. Entre las empresas que más destacan en Biotecnología, Productos farmacéuticos y Química aparecen algunas EMAs japonesas. Finalmente, entre las que más destacan en innovación de diseño aparecen algunas empresas que no figuraban entre las más importantes en relación a la innovación técnica.

Esta información sugiere, aunque desde luego en forma muy preliminar, que las multinacionales pueden tender a competir con diferentes herramientas técnicas o de diseño. 


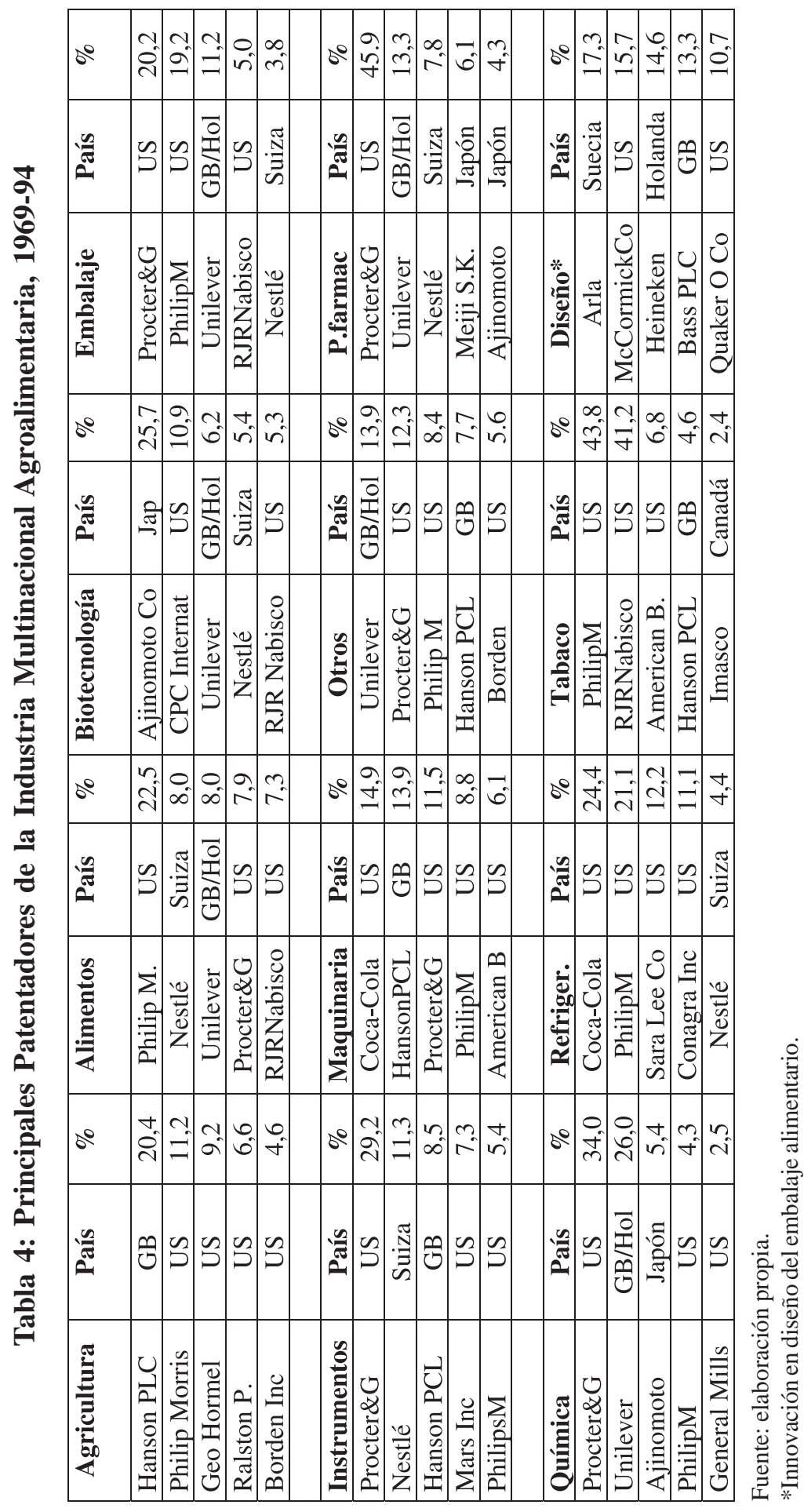




\section{Conclusiones}

En este trabajo hemos investigado la cartera tecnológica de un grupo de 90 empresas multinacionales agroalimentarias seleccionadas entre las 100 mayores del mundo. Para ello, hemos analizado 18.611 patentes de utilidad (técnicas) y 1.359 de diseño que se les otorgaron en los EEUU en el periodo 1969-94.

Las multinacionales no sólo producen una enorme cantidad de innovaciones de Alimentos sino que también son innovadoras en una multiplicidad de campos técnicos aparentemente ajenos a la agroindustria. Aparte el campo técnico de Alimentos, los campos donde la multinacional agroalimentaria patenta más, en promedio, son los de Química y Otras patentes.

Las EMAs innovadoras en el campo de los Alimentos también tienden a serlo en cuatro campos no alimentarios: Biotecnología, Química, Maquinaria y Otros. Estos resultados son estadísticamente significativos y robustos. La asociación entre las variables se confirma en diversos grupos de multinacionales de diferente país de origen y cuando se extraen de la muestra a los casos atípicos (posibles conglomerados dada su alta producción de patentes no alimentarias). Este resultado sugiere que la producción de patentes en estos campos no técnicos por parte de estas multinacionales no obedece sólo a que la muestra contenga unos pocos conglomerados, sino que cada empresa, sea cual sea su naturaleza, combina innovaciones de Alimentos con un número variable de innovaciones no alimentarias. Inclusive las EMAs escasamente innovadoras en Alimentos también producen un pequeño número de innovaciones no alimentarias.

Este resultado apoya la idea que, por lo regular, las EMAs no son colecciones inconexas de diferentes innovaciones tecnológicas, como a veces se aduce, sino que generan conocimientos de diverso tipo con un propósito relativamente unificado. Ese propósito puede ser variado: producir alimentos, elaborar inputs para otros procesadores o bien aprovechar con miras industriales los subproductos de la cadena agroalimentaria. Esos conocimientos no se concretan necesariamente en la producción de equipamientos e inputs para la producción agroalimentaria. Dado el pequeño número de patentes que las EMAs producen en determinados campos no alimentarios, es más probable que sean el fruto ocasional de un proceso interno de aprendizaje cuyo objetivo es mantener a la empresa al tanto de nuevas técnicas en campos afines o ayudarla a desarrollar relaciones más fructífera con sus proveedores. En gran medida, por lo tanto, la actividad patentadora de las EMAs en campos no alimentarios podría proceder de un esfuerzo deliberado y sistemático para adquirir información.

Diferentes multinacionales y grupos de multinacionales, según su país de origen, 
parecen competir sobre la base de diferentes maneras de combinar dichos conocimientos. De acuerdo con esas combinaciones, las empresas japonesas, por ejemplo, parecen posicionadas en mercados de inputs biotecnológicos mientras que las occidentales se especializan en aspectos del embalaje, más importantes en el mercado de consumo final.

Este estudio sugiere que no sólo la intensidad de la actividad innovadora sino también las piezas componentes de su esfuerzo de I+D hacen de las EMAs un oponente formidable para sus rivales. Sus ventajas competitivas en materia tecnológica derivan no sólo de su capacidad de innovar, como ha sostenido siempre la teoría económica (Caves, 1996), sino también de combinar intramuros diferentes tipos de conocimientos técnicos. Frente a ellas, la empresa nacional puede encontrarse en inferioridad de condiciones desde el punto de vista técnico. En muchos países, como en el Brasil, sólo un reducidísimo número de empresas nacionales agroalimentarias son innovadoras y, de ellas, un número aún menor es capaz de integrar intramuros su propia cadena tecnológica (Cabral, 2000).

\section{Agradecimientos}

Una primera versión de este trabajo fue presentada al "XXVII Encontro ANPAD” donde obtuvo el Primer Premio en el Área de “Gestión de los Agronegocios”. Los autores agradecen a los Prof. Antonio Padula, Jean-Philippe P. Révillion y demás participantes sus comentarios y sugerencias. De igual modo, Oscar Alfranca agradece la financiación recibida a través del Proyecto No SGR2001160 de la Direcció General de Recerca, Departament d’Universitats, Recerca i Societat de la Informació, de la Generalitat de Catalunya.

\section{Notas}

\footnotetext{
${ }^{1}$ Según información de AGRODA, en el Brasil conviven las filiales de 50 de las 100 mayores EMAs del mundo.

${ }^{2}$ El campo técnico no es un concepto equivalente al de industria. Como se verá más adelante, las empresas agroalimentarias, por ejemplo, pueden patentar inventos en campos técnicos correspondientes a la química, la biotecnología, etc. aunque no produzcan este tipo de productos.

${ }^{3}$ Las fuentes utilizadas por el IAMM son el “Moody’s Industrial Manual”, el "Fortune Directory of the 500 largest US and 500 largest non-US corporations", el "Dossier 5.000" de las mayores multinacionales europeas de "Le Nouvel Economiste”, Dun y Branstreet y los informes anuales de las empresas. Todas las EMAs incluídas en esta base de dato tienen ventas anuales de al menos 1 billón de US\$, destinan al menos el 50\% de sus ventas a alimentos procesados y poseen, al menos, una filial internacional .
} 
${ }^{4}$ Todas ellas tienen filiales en EEUU, por lo que se supone que tienen interés en proteger su propiedad intelectual en dicho mercado, por lo demás el mayor productor mundial de tecnología agroalimentaria

${ }^{5}$ La información procede de la US Patent Office (USPTO) que la publica, desde 1975 solamente, on line (http://www.uspto.gov). Esta información no aparece por casa matriz de las EMAs. Recolectarla supone agrupar los datos correspondientes a diversas filiales patentadoras, que es al nivel que viene la información. En grandes empresas como Nestlé o Unilever, aquellas pueden ser muy numerosas. Luego, a través del Who Owns Whom, que contiene el nombre de las filiales de las mayores empresas mundiales, se procede a consolidar la información de patentes al nivel de la empresa. Los datos de USPTO no permiten distinguir entre innovación de producto y de proceso.

${ }^{6}$ Estos campos corresponden a 332 clases de USPTO que es imposible detallar aquí por problemas de espacio. El campo técnico Alimentos, por ejemplo, comprende tres clases diferentes.

${ }^{7}$ Utilizamos correlaciones no paramétricas porque el estadístico de Jarque-Bera rechaza la hipótesis de normalidad para todas las variables analizadas.

${ }^{8}$ Como ya se ha advertido anteriormente los campos técnicos no equivalen a líneas productivas. Como se verá más adelante, las empresas que patentan, por ejemplo, en Química no tienen necesariamente subsidiarias en dicha rama industrial.

Artigo recebido em 13.10.2003. Aprovado em 22.12.2003.

\section{Bibliografía}

ACS, Z. J.;

AUDRETSCH, D. B.

Patents as a measure of innovative activity. WZB, Berlin: p. 1-13. 1989.

\section{ALFRANCA, O.; \\ RAMA, R.; \\ VONTUNZELMANN, N.}

A patent analysis of global food and beverage firms: the persistence of innovation. Agribusiness. An International Journal, v. 18n. 3. 2002.

ALFRANCA, O;

RAMA, R.;

VONTUNZELMANN, N.

Competitive behaviour, design and technical innovation in food and beverage multinationals. International Journal of Biotechnology, co-edition with IJTM, Special issue on Innovation in Food and Beverages, and Biotechnology, v5, n3/4.p.222-248, 2004.

\section{ANASTASSOPOULOS, G;}

RAMA, R.

The performance of multinational agribusinesses: effects of product and geographical diversification. In: Rama, R. (Ed.). Multinational agribusinesses. N.Y. forthcoming: Haworth Press Inc, 2004.

ARCHIBUGI, D.;

PIANTA, M.

Specialization and size of technological activities in industrial countries: The analysis of patent data. Research Policy, v. 21, p. 79-93, 1992. 
BELIK, W.

The Food Industry in Brazil: Towards a Restructuring? Institute of Latin American Studies Research Papers. p.1-42, 1994.

BIJMAN, W. B.;

ENZING, C. M.

Biotechnology and vertical coordination in the agrofood chain: a case study of the Dutch potato chain. Science and Public Policy, v. 22 n.6, 1995.

BOUND, J.;

CUMMINS, C.;

GRILICHES, Z;

HALL, B. H.;

JAFFE, A.

Who does R\&D and who patents? In: GRILICHES, Z. (Ed.), R\&D, Patents, and Productivity. NBER, University of Chicago Press, 1984.

BYÉ, P.

The food industry: still a craft industry? International Journal of Technology Management, v. 16, n. 7, 1998.

CABRAL, J.E. O.

Pattern and determinants of technological innovation in the Brazilian food industry. Unpublished PhD Thesis, University of Reading, Reading. 2000.

CAVES, R.E.

Multinational enterprise and economic analysis. 2. ed. Cambridge: University Press, 1996.
CHRISTENSEN, J. L.;

RAMA, R.;

VONTUNZELMANN, N.

Study on innovation in the European Food Products and Beverages Industry: 145. EIMS/SPRINT Brussels: The European Commission, 1996.

\section{COHEN, W. M.;}

LEVINTHAL, D.A.

Innovation and learning: the two faces of R\&D. Economic Journal, v.9, p. 569-596, 1989.

\section{CONNOR, J. M.}

Food product proliferation: a market structure analysis. American Journal of Agricultural Economics, 1981.

DING J. Y;

CASWELL, J.A.

Changes in Diversification among very Large Food Manufacturing Firms in the 1980s. Agribusiness: An International Journal, v. 11, n. 6, p. 553-563. 1995.

DOSI, G;

TEECE, D.;

WINTER, S. G.

Toward a theory of corporate coherence: preliminary remarks. In: DOSI, G.; GIANNETTI, R.; TONINELLI, P.A. (Eds.). Technology and enterprise in a historical perspective. Oxford: Clarendon Press. p.185-211, 1992.

DUNNING, J.H.

The globalisation of business. London-NY: Routledge, 1993. 
FAGERBERG J.

A technology gap approach to why growth rates differ. Research Policy, v. 16, 1987, p. 87-99.

FARINA, E. M. M. Q.;

SANTOS VIEGAS, C. A.

Multinational Firms in the Brazilian Food Industry. In: Rama, R. (Ed.). Multinational agribusinesses. N.Y.: Haworth Press, 2004.

\section{FREEMAN, C.}

The economics of technical change. Cambridge Journal of Economics, v. 18, p. 463-514, 1994.

\section{GE.S.T.}

Grappes technologiques. Les nouvelles stratégies d'entreprise. Paris: McGraw-Hill, 1986.

GALIZZI, G;

VENTURINI, L.

Product innovation in the food industry: Nature, characteristics and determinants. In G. Galizzi \& L. Venturini (Eds.), Economics of Innovation: The Case of Food Industry, Heidelberg: Physica-Verlag. 1996.

GONARD, T;;

GREEN, R.H.;

MALERBE, A.;

REQUILLART, $\mathrm{V}$.

Changement technique et stratégie des acteurs dans le secteur de la chimie du sucre. INRA, Economie et Sociologie Rurales, 7 (Special issue on "Changement technique et restructuration de l'industrie agroalimentarie en Europe”): 143-158, 1991.
OECD.

Impact of Multinational Enterprises on National Scientific and Technical Capacities. Paris: OECD, 1979.

PATEL, P.

Localised production of technology for global markets. Cambridge Journal of Economics, v. 19, p. 141153, 1995.

\section{PATEL, P.;}

PAVITT, K.

Large firms in the production of the world's technology: an important case of 'non-globalisation. Journal of International Business Studies, v. 22, p. 1-21, 1991.

The technological competencies of the world's largest firms: complex and path-dependent, but not much variety. Research Policy v. 26, p. 141156, 1997.

\section{PETRONI, A.}

Patterns of technological innovation in subcontracting firms: an empirical study in the food machinery industry. European Journal of Innovation Management, v. 3., n. 1, p. 15-26, 2000.

RAMA, R.

El entorno tecnológico de la empresa alimentaria. INRA Economie et Sociologie Rurales. v. 7 (Special issue on: Changement Technique et restructuration de l'industrie agroalimentaire en Europe), p. 59-93. Paris, 1991. 
An empirical study on sources of innovation in the international Food and Beverage industry. Agribusiness: An International Journal, v.12, p. 123-134, 1996.

RASTOIN, J. L.;

GHERSI, G;

PÉREZ, R.;

TOZANLI, S.

Structures, performances et stratégies des groupes agro-alimentaires multinationaux. Montpellier:

AGRODATA, 1998.

ROSENBERG, N.

Inside the Black Box: Technology and

Economics. Cambridge. 1982.
SOETE, L.

The impact of technological innovation on international trade patterns: The evidence reconsidered. Research Policy, v. 16, p. 101-130, 1987.

\section{TOZANLI, S.}

Capital concentration among the food multinational enterprises and development of the world's agro-food system. Int. J. Technology Management, v.16, n. 7, p. 695-710, 1998.

\section{VONTUNZELMANN, G. N.}

Localized technological search and multi-technology companies. Economics of Innovation and New Technology, v. 6, p. 231-255, 1998. 